\title{
Identification of Two Independent COL5A1 Variants in Dogs with Ehlers-Danlos Syndrome
}

\author{
Anina Bauer 1,2, John F. Bateman 3,4® ${ }^{3}$, Shireen R. Lamandé 3,5, Eric Hanssen 4,6, \\ Shannon G.M. Kirejczyk ${ }^{7} \mathbb{D}$, Mark Yee ${ }^{8}$, Ali Ramiche ${ }^{9}$, Vidyha Jagannathan ${ }^{1,2}{ }^{\mathbb{D}}$, \\ Monika Welle ${ }^{4,10}$, Tosso Leeb ${ }^{1,2}$ (D) and Fiona L. Bateman ${ }^{3,11, *}$ \\ 1 Institute of Genetics, University of Bern, 3012 Bern, Switzerland; anina.bauer@vetsuisse.unibe.ch (A.B.); \\ vidhya.jagannathan@vetsuisse.unibe.ch (V.J.); tosso.leeb@vetsuisse.unibe.ch (T.L.) \\ 2 Dermfocus, University of Bern, 3012 Bern, Switzerland \\ 3 Murdoch Children's Research Institute, Parkville, Victoria 3052, Australia \\ john.bateman@mcri.edu.au (J.F.B.); shireen.lamande@mcri.edu.au (S.R.L.) \\ 4 Department of Biochemistry and Molecular Biology, University of Melbourne, Parkville, Victoria 3010, \\ Australia; ehanssen@unimelb.edu.au (E.H.); monika.welle@vetsuisse.unibe.ch (M.W.) \\ Department of Paediatrics, University of Melbourne, Parkville, Victoria 3010, Australia \\ 6 Melbourne Advanced Microscopy Facility, Bio21 Molecular Science and Biotechnology Institute, \\ The University of Melbourne, Parkville, Victoria 3010, Australia \\ 7 College of Veterinary Medicine, Department of Pathology, The University of Georgia, Athens, GA 30602, \\ USA; skirejc@emory.edu \\ 8 Torquay Animal Hospital, Torquay, Victoria 3228, Australia; info@torquayvets.com.au \\ 9 Veterinary Practice, Agadir 86603, Morocco; ali.ramiche@umontreal.ca \\ 10 Institute of Animal Pathology, University of Bern, 3012 Bern, Switzerland \\ 11 College of Veterinary Medicine, Small Animal Medicine and Surgery, The University of Georgia, \\ Athens, GA 30602, USA \\ * Correspondence: fibatema@gmail.com; Tel.: +1-706-542-3221
}

Received: 10 July 2019; Accepted: 16 September 2019; Published: 21 September 2019

\begin{abstract}
The Ehlers-Danlos syndromes (EDS) are a heterogeneous group of heritable disorders affecting connective tissues. The mutations causing the various forms of EDS in humans are well characterized, but the genetic mutations causing EDS-like clinical pathology in dogs are not known, thus hampering accurate clinical diagnosis. Clinical analysis of two independent cases of skin hyperextensibility and fragility, one with pronounced joint hypermobility was suggestive of EDS. Whole-genome sequencing revealed de novo mutations of COL5A1 in both cases, confirming the diagnosis of the classical form of EDS. The heterozygous COL5A1 p.Gly1013ValfsTer260 mutation characterized in case 1 introduced a premature termination codon and would be expected to result in $\alpha 1(\mathrm{~V})$ mRNA nonsense-mediated mRNA decay and collagen $V$ haploinsufficiency. While mRNA was not available from this dog, ultrastructural analysis of the dermis demonstrated variability in collagen fibril diameter and the presence of collagen aggregates, termed 'collagen cauliflowers', consistent with COL5A1 mutations underlying classical EDS. In the second case, DNA sequencing demonstrated a p.Gly1571Arg missense variant in the COL5A1 gene. While samples were not available for further analysis, such a glycine substitution would be expected to destabilize the strict molecular structure of the collagen $\mathrm{V}$ triple helix and thus affect protein stability and/or integration of the mutant collagen into the collagen $\mathrm{V} /$ collagen I heterotypic dermal fibrils. This is the first report of genetic variants in the COL5A1 gene causing the clinical presentation of EDS in dogs. These data provided further evidence of the important role of collagen $\mathrm{V}$ in dermal collagen fibrillogenesis. Importantly, from the clinical perspective, we showed the utility of DNA sequencing, combined with the established clinical criteria, in the accurate diagnosis of EDS in dogs.
\end{abstract}


Keywords: Collagen; Cutaneous asthenia; Dog; Canis familiaris; Dermatology; Skin; Whole-genome sequencing; Precision medicine; Rare disease; Genodermatosis

\section{Introduction}

The Ehlers-Danlos syndromes (EDS) are a heterogeneous group of heritable disorders affecting connective tissue. In humans, variable clinical manifestations affecting primarily the skin, joints, ligaments, blood vessels, and internal organs have been reported. EDS has also been seen in many animal species, including horses [1], mink [2], rabbits [3], dogs [4-14], and cats [15-18].

Abnormal collagen fibril formation is the hallmark of several types of human EDSs, including the classical form [19]. In the classical form of EDS, mutations in COL5A1 and COL5A2, which encode the $\alpha 1$ - and $\alpha 2$-chain of type $\mathrm{V}$ collagen, have been reported [20-26]. Type $\mathrm{V}$ collagen is a quantitatively minor fibril-forming collagen. Several isoforms exist, but the most widely distributed form is the $[\alpha 1(\mathrm{~V})]_{2} \alpha 2(\mathrm{~V})$ heterotrimer that co-assembles with type I collagen into heterotypic type I/V collagen fibrils in the extracellular matrix. Type $V$ collagen is thought to regulate the diameter of these fibrils by retention of its large N-propeptide domain, which projects above the surface of the collagen fibril [27]. In humans, mutations in COL5A1 and COL5A2 lead to characteristic collagen abnormalities in the skin with variability in collagen fibril diameter and the presence of collagen aggregates, termed 'collagen cauliflowers' [28]. Abnormal collagen structure and fibril formation, in turn, contribute to the clinical signs seen in classic EDS, including hyperextensible skin, generalized joint hypermobility, and generalized connective tissue fragility [29].

We reported here three dogs with two different genetic variants in the COL5A1 gene affecting collagen synthesis and structure, analogous to classical EDS in human patients. Furthermore, we described histopathological and ultrastructural findings associated with the COL5A1 variants in these animals. Collagen protein analysis of one affected individual was also performed.

\section{Materials and Methods}

\subsection{Ethics}

The collection of blood and skin samples from case 1 and the related controls (FB and MY) were approved by the Murdoch Children's Research Institute Animal Ethics Committee (Approval \# A815). Blood and skin samples for cases 2 and 3 (RA) were collected as part of routine veterinary diagnostic assessment. For biopsy samples, animals were sedated with diazepam $0.5 \mathrm{mg} / \mathrm{kg}$ IV (Diapharm ${ }^{\circledR} 10 \mathrm{mg} / \mathrm{mL}$, Pharma5, Morocco) and ketamine $6 \mathrm{mg} / \mathrm{kg}$ IV (Imalgene ${ }^{\circledR} 1000$, Merial, France) via peripheral catheter to achieve a moderate level of sedation. Biopsies were collected as per standard techniques, and the animals recovered uneventfully. Taking blood samples from healthy control dogs for genetic analysis was approved by the "Cantonal Committee For Animal Experiments" (Canton of Bern; permit 75/16).

\subsection{Histopathology}

\subsubsection{Case 1}

One $6 \mathrm{~mm}$ diameter punch biopsy was obtained from the dorsum of the 6-month-old Labrador, fixed in 10\% neutral-buffered formalin, processed routinely, and embedded in paraffin wax. Four-to-five-micron-thick sections were stained with hematoxylin and eosin (H\&E), Masson's Trichrome (MT), and Verhoeff van Gieson (VVG) stains at the University of Georgia, College of Veterinary Medicine Histology Laboratory. As a control, a punch biopsy was obtained from the abdominal region of a 10-month-old, intact male, Labrador dog, fixed in formalin, processed routinely, and stained with H\&E, MT, and VVG for comparison. Slides were reviewed by a board-certified veterinary pathologist (SK). 


\subsubsection{Cases 2 and 3}

One $8 \mathrm{~mm}$ punch biopsy from the lateral thorax was investigated histologically. The biopsy was fixed in $10 \%$ buffered formalin and processed routinely. The slide was stained with hematoxylin and eosin (H\&E) prior to histopathological examination by a board-certified veterinary pathologist (MW).

\subsection{Transmission Electron Microscopy (TEM)}

For electron microscopy, dermal samples were fixed in $0.1 \mathrm{M}$ sodium cacodylate containing $2.5 \%$ glutaraldehyde; after rinsing, the samples were post-fixed in $1 \%$ aqueous osmium tetroxide, dehydrated in an alcohol series, and embedded in Epon 812. Seventy-nanometer ultrathin sections were cut and observed on a Tecnai F30 (FEI, Eindhoven, NL, USA) with an extraction voltage of $200 \mathrm{kV}$. Micrographs were taken using a Gatan UltraScan 1000 (Gatan, Pleasanton, CA, USA).

\subsection{Biochemical Analysis of Dermis}

Full-thickness dermal biopsies were diced with a scalpel and defatted by gentle shaking with cold chloroform:methanol $(2,1)$ for $24 \mathrm{~h}$. Tissue was washed with cold methanol and dried under vacuum. Dried tissue was re-hydrated in $50 \mathrm{mM}$ Tris/ $\mathrm{HCl}$, pH 7.5 containing $0.15 \mathrm{M} \mathrm{NaCl}$. To assist in the uniformity of extraction, the re-hydrated tissue was snap-frozen and powered under liquid nitrogen. For collagen analysis, a sequential extraction protocol was used to extract the successively more cross-linked collagen matrix, as previously described [30]. The freeze-milled dermis was first extracted with $0.15 \mathrm{M} \mathrm{NaCl}, 50 \mathrm{mM}$ Tris- $\mathrm{HCl}$ buffer for $24 \mathrm{~h}$ at $4{ }^{\circ} \mathrm{C}$ to remove the soluble collagens followed by extraction with $4 \mathrm{M}$ guanidine hydrochloride $(\mathrm{GuHCl})$ in Tris- $\mathrm{HCl}$ buffer $(\mathrm{pH} 7.4)$, then $0.5 \mathrm{M}$ acetic acid, and finally digestion with pepsin at $100 \mu \mathrm{g} / \mathrm{mL}$ in $0.5 \mathrm{M}$ acetic acid to sequentially extract the successively more cross-linked and thus mature collagen matrix. To enrich for collagen $\mathrm{V}$, selective salt precipitation of portions of the pepsin extracted collagen was performed [31]. Collagen chains in each extract were analyzed on SDS-gradient polyacrylamide gels (3-8\%, Tris-Acetate Gel, Novex Life Technologies, Carlsbad, CA, USA), visualized by Coomassie Brilliant Blue staining and quantified, as described previously [30]. Pepsin-soluble collagen from rat tail tendon was run on each gel as a standard for quantitation.

\subsection{DNA Extraction and Whole-Genome Sequencing}

Genomic DNA was isolated from EDTA blood samples of the investigated dogs. For case 1 and case 2, an Illumina PCR-free TruSeq fragment library with $\sim 390 \mathrm{bp}$ insert size was prepared. We collected $\sim 153$ and 211 million $2 \times 150$ bp paired-end reads or $\sim 18 \times$ and $\sim 25 \times$ coverage on an Illumina HiSeq 3000 instrument for cases 1 and 2, respectively. The reads were mapped to the CanFam3.1 dog reference genome assembly and aligned using Burrows-Wheeler Aligner (BWA) version 0.7.5a [32] with default settings. The generated SAM file was converted to a BAM file, and the reads were sorted by coordinate using SAMtools [33]. Picard tools (http://sourceforge.net/projects/picard/) were used to mark PCR duplicates. To perform local realignments and to produce a cleaned BAM file, we used the Genome Analysis Tool Kit (GATK version 2.4.9, 50) [34]. GATK was also used for base quality recalibration with canine dbsnp version 139 data as a training set. The sequence data were deposited under the study accession PRJEB16012 and sample accessions SAMEA104091568 (case 1), and SAMEA4867923 (case 2), at the European Nucleotide Archive. Additionally, we used 356 additional whole-genome sequences as controls, which were either publicly available [35], produced during other projects of our group or contributed by members of the Dog Biomedical Variant Database Consortium.

Putative SNVs were identified in each of the 358 samples individually using GATK HaplotypeCaller in gVCF mode [36]. Subsequently, all sample gVCF files were joined using Broad GenotypeGVCFs walker (-stand_emit_conf 20; -stand_call_conf 30). Filtering was performed using the variant filtration module of GATK using the following standard filters: SNPs: Quality by Depth: QD <2; Mapping quality: MQ < 40; Strand filter: FS > 60; MappingQualityRankSum: MQRankSum <-12.5; ReadPosRankSum < -8 and INDELs: Quality by Depth: QD $<2$; Strand filter: FS $>200$. The functional effects of the called variants were predicted using SnpEFF software [37] together with the NCBI annotation release 105 on 
CanFam 3.1. Identified sequence variants were confirmed by Sanger sequencing of PCR amplicons. Targeted genotyping of additional dogs was also done by Sanger sequencing.

\section{Results}

\subsection{Clinical Assessment}

\subsubsection{Case 1}

A 4-month-old male intact Labrador was examined for several small skin lacerations of the distal limbs, hock, and tail base. On physical examination, the dog was found to be clinically healthy, with normal skeletal development. Several seroma-like swellings were noted in the regions of skin trauma (hocks, tail base). Aspirates taken from these swellings revealed moderate numbers of erythrocytes with fewer neutrophils and macrophages with proteinaceous debris. These findings were consistent with a seroma or hematoma formation, and the dog was treated with amoxycillin/clavulanic acid (Clavulox ${ }^{\circledR}$, Zoetis, Ryde, Australia) and firocoxib (Previcox ${ }^{\circledR}$, Zoetis, Ryde, Australia).

Two months after initial presentation, the dog was again examined for ongoing seroma formation and joint abnormalities. On physical examination, evidence of generalized joint hyperextensibility with a range of motion greater than $180^{\circ}$ (Figure 1) and skin hyperextensibility (Figures 2 and 3 ) and fragility was noted. The dog had a skin extensibility test [38] of $23.5 \%$ (normal $<14.5 \%$ ) [39]. A complete blood count revealed mild anemia of $4.87 \times 10^{12} / \mathrm{L}$ (5.5-8.5), HCT $0.33 \mathrm{~L} / \mathrm{L}(0.37-0.55), \mathrm{Hb} 113 \mathrm{~g} / \mathrm{L}$ (120-180), and a normal white cell count. Serum biochemistry showed a mild hypoalbuminemia $21 \mathrm{~g} / \mathrm{L}$ (28-42) and hypoproteinemia $47 \mathrm{~g} / \mathrm{L}$ (54-78). Other serum abnormalities included a moderate hyperphosphatemia, mild elevation in ALP, and decrease in creatinine that were all considered within normal limits for a young dog. At this time, due to the ongoing joint hypermobility and skin lacerations with the suspicion of genetic collagen disease, the owners elected to humanely euthanize the dog.

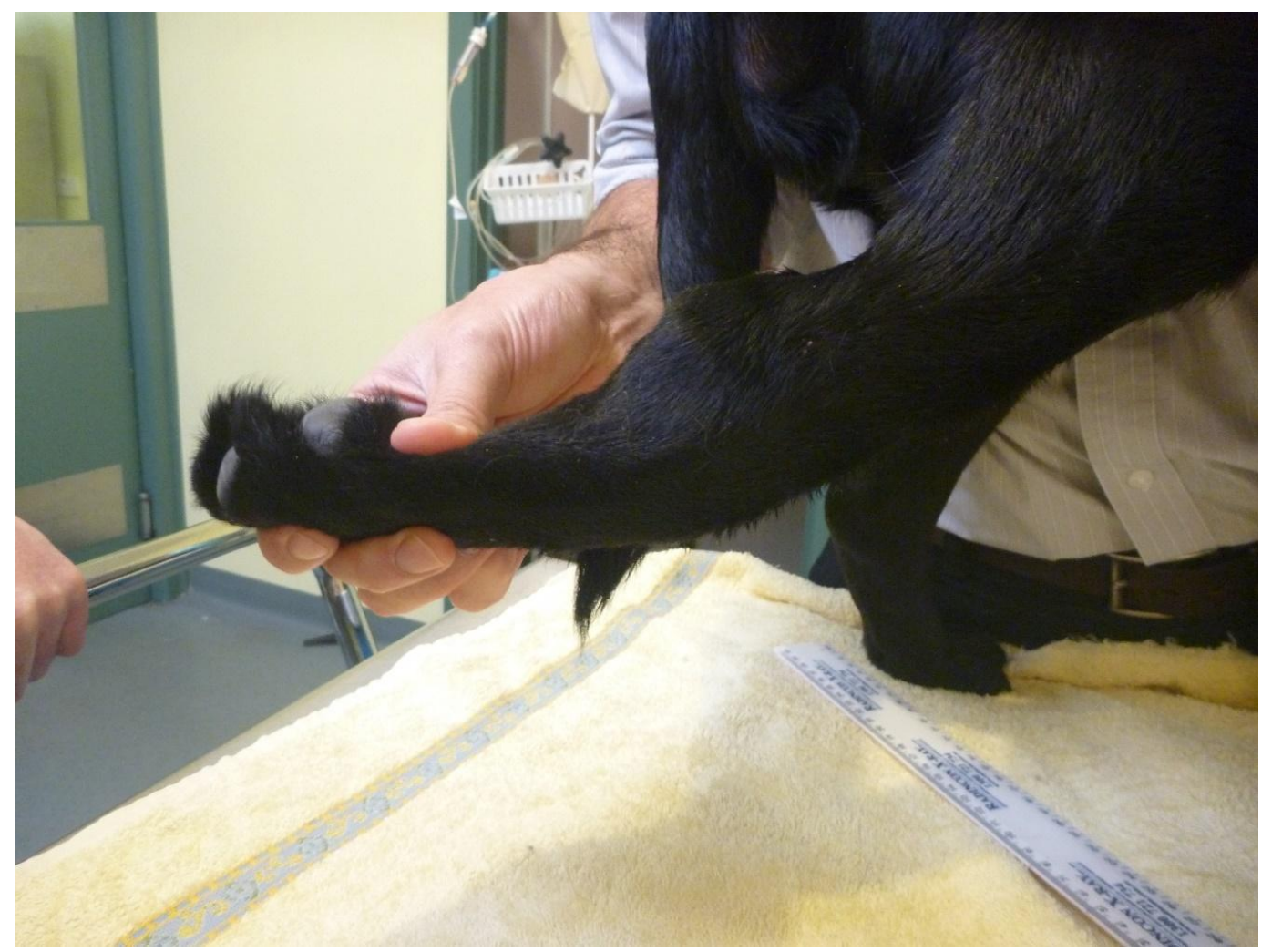

Figure 1. Case 1, Joint hypermobility of the tarsus and metatarsus. 


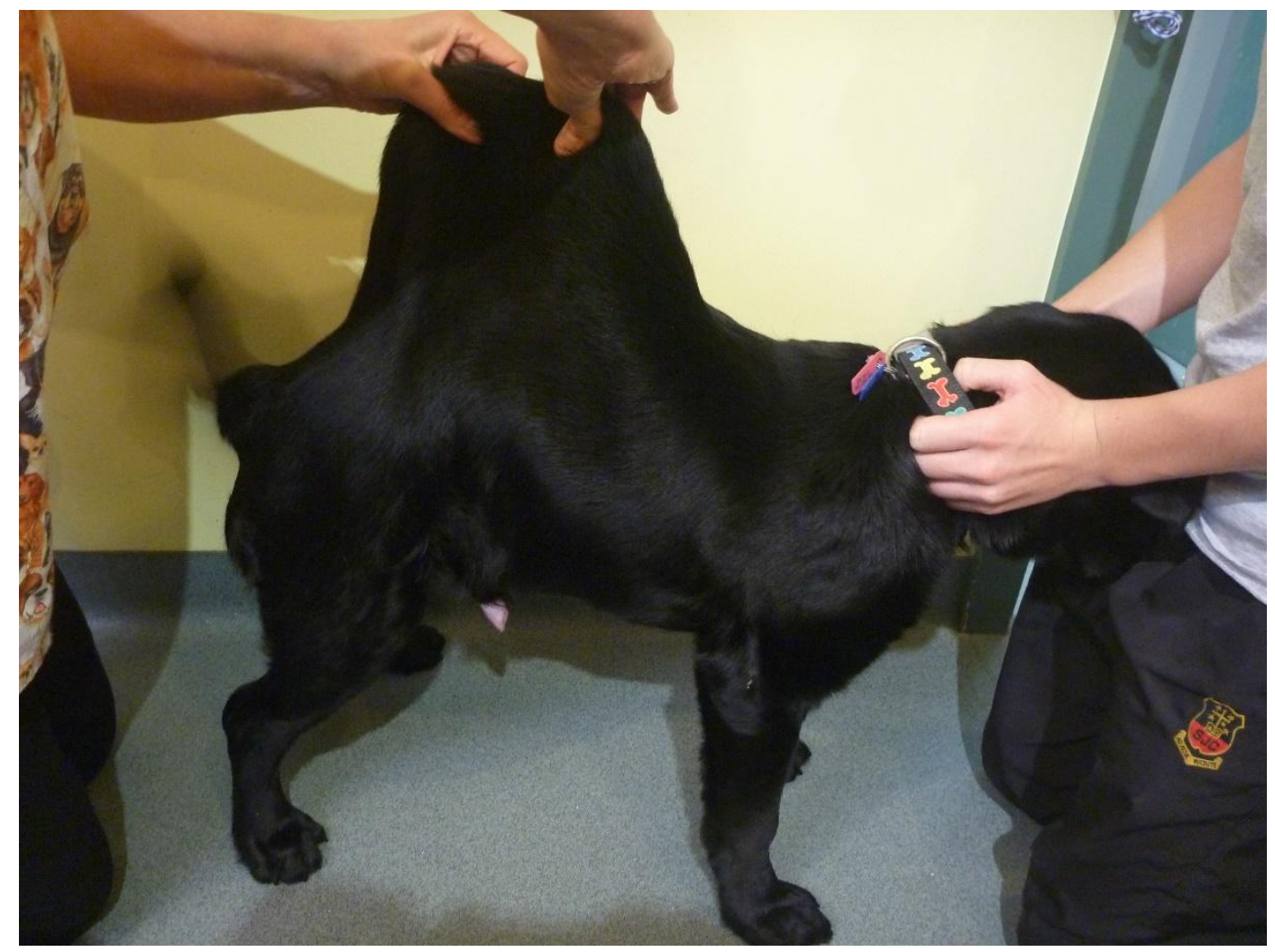

Figure 2. Case 1, Skin hyperextensibility over the dorsum.

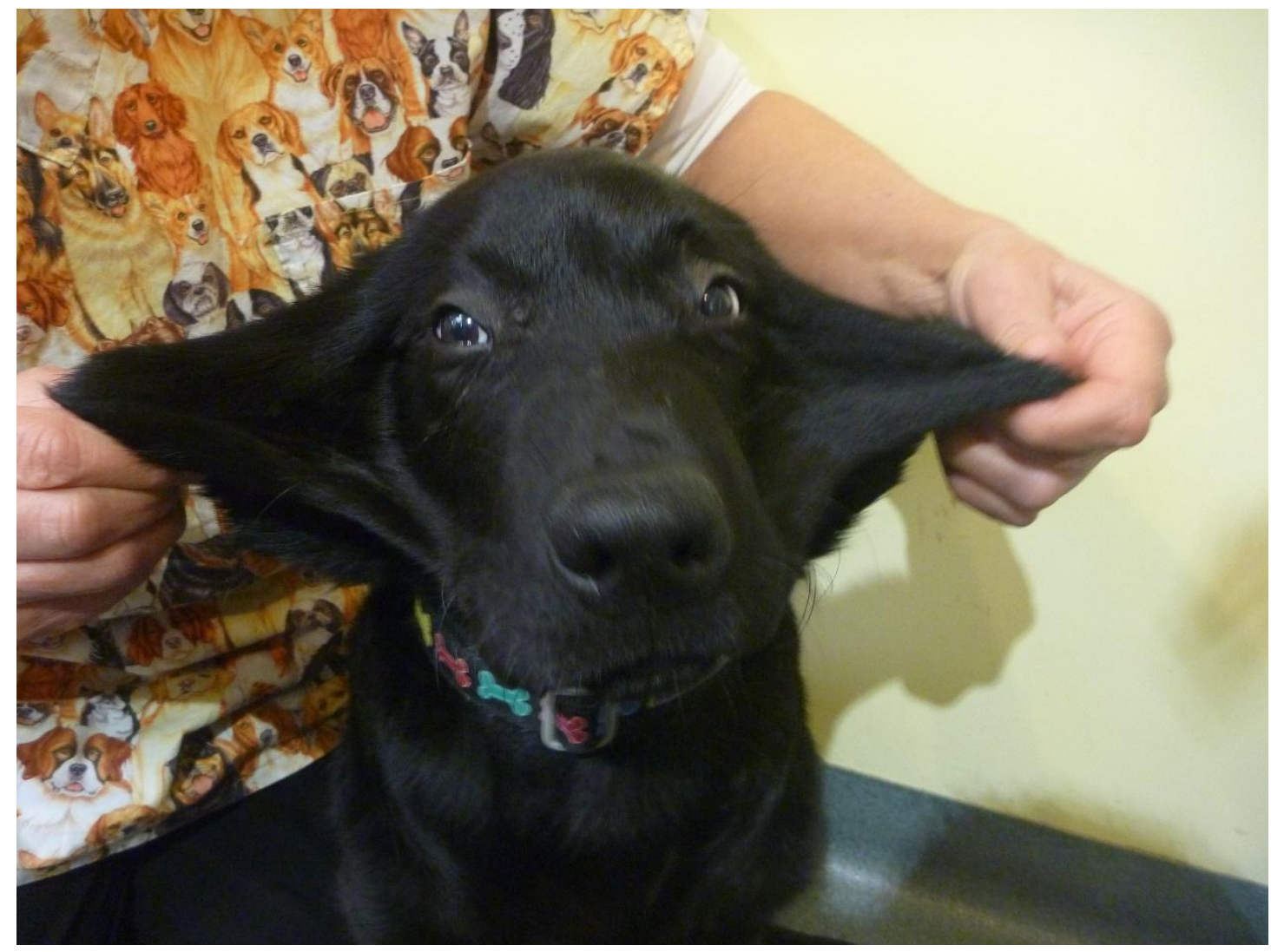

Figure 3. Case 1, Skin hyperextensibility of the face. 


\subsubsection{Cases 2 and 3}

Two young adult mixed breed dogs were presented for hematomas and skin lacerations after mild trauma. The dogs (siblings) were adopted from an animal shelter in the southeast region of Morocco. The prior clinical history and onset of clinical signs were unknown, though it was noted that the dam was unaffected. On physical examination, the dogs were found to be clinically healthy, with normal skeletal development. Bruising and skin lacerations were noted on the carpi with oozing, hemorrhagic zones of 3-4 cm in size with disruption of the skin barrier (splitting of the skin) in these regions (case 2). Bruising and wounds were noted to occur after only mild trauma. There was hyperelasticity of the elbows (Figure 4) and tarsi as well as the skin of the neck (Figure 5) and flank (case 2 and 3), with evidence of the previous scarring on the neck (case 2). The rest of the clinical exam was unremarkable for both dogs. The presence or absence of joint hypermobility was not described in the medical records.

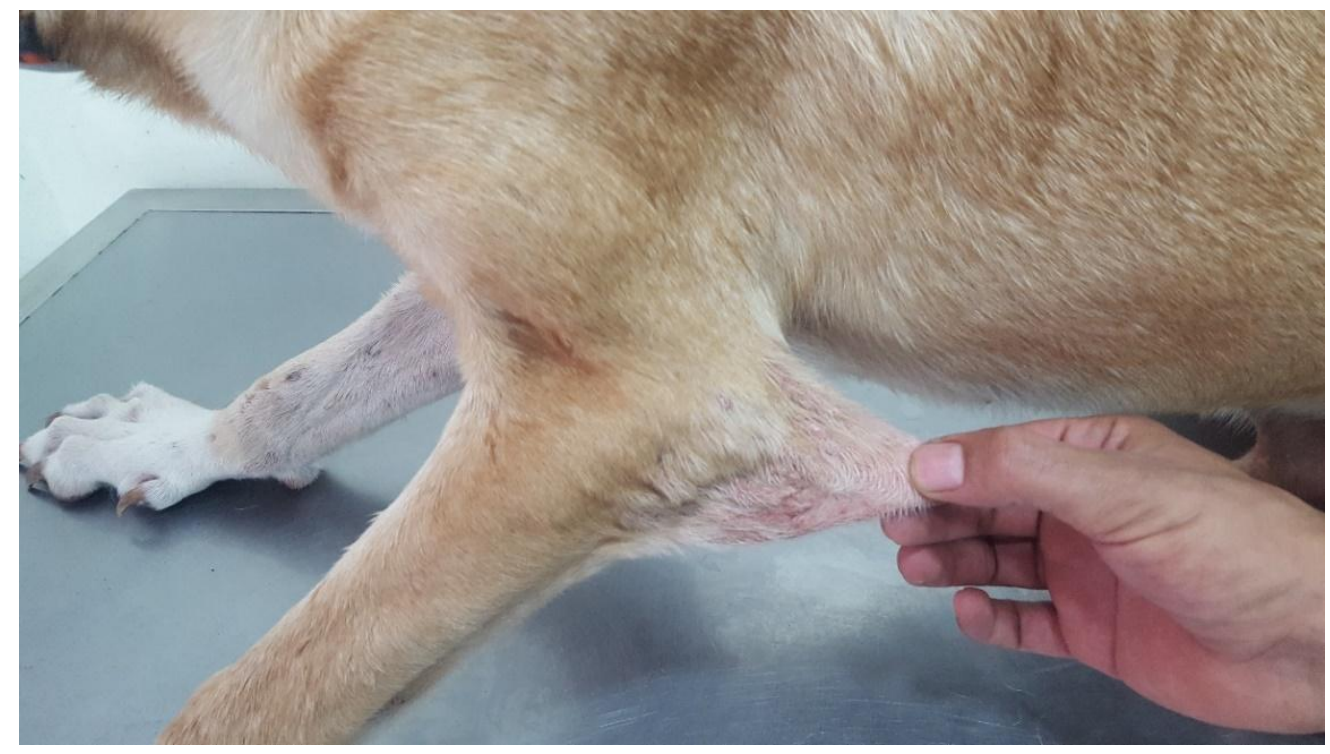

Figure 4. Case 2, Skin hyperextensibility over the elbow.

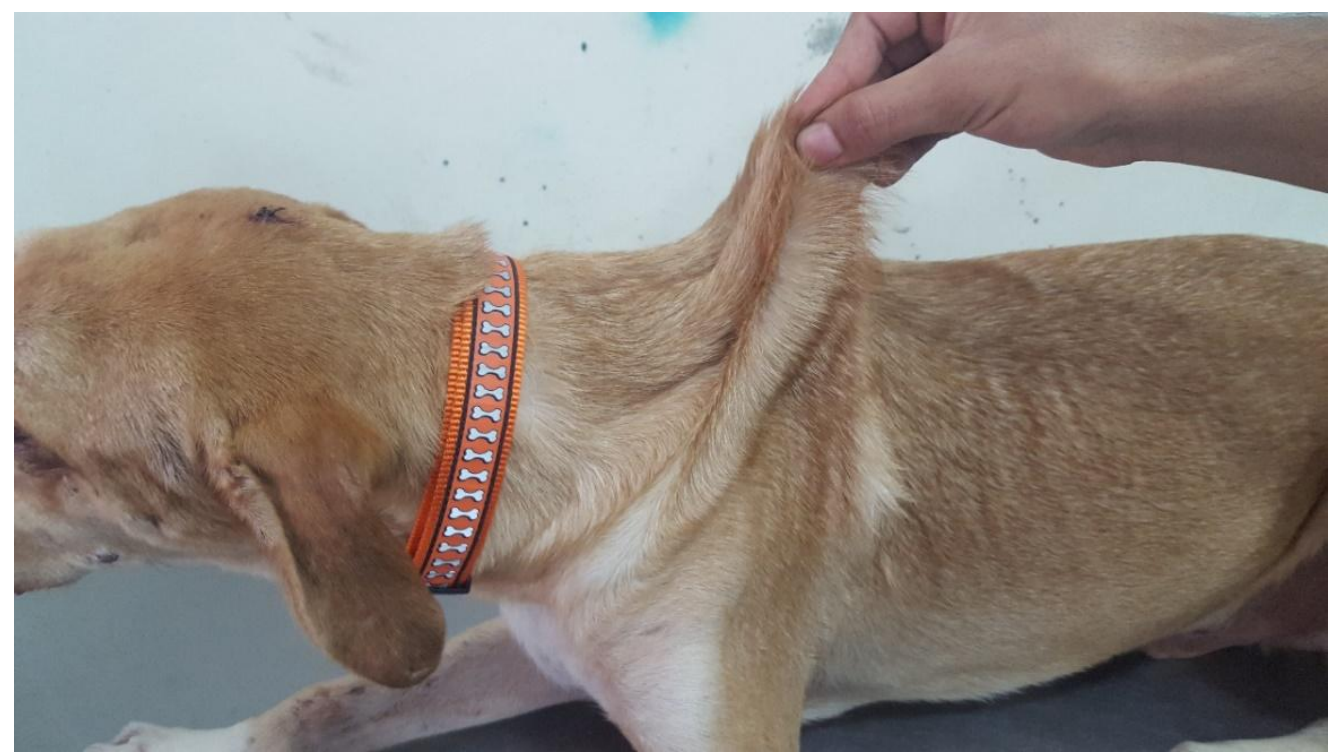

Figure 5. Case 2, Skin hyperextensibility over the dorsum. 
On routine screening of blood work for case 2 , the hematocrit was normal at $0.48 \mathrm{~L} / \mathrm{L}(0.37-0.55)$ and total solids $54 \mathrm{~g} / \mathrm{L}$ (54-78). While skin biopsy of case 2 and 3 was performed, no further diagnostic testing was undertaken. The outcome of these dogs is currently not known.

\subsection{Histopathology}

\subsubsection{Case 1}

Histological examination of skin from the 4-month-old Labrador revealed dermal collagen fiber abnormalities, characterized by fiber disarray, variability in fiber diameter, and multifocal fiber clumping (Figure 6, Panels A and B). Collagen clumps stained hypereosinophilic on H\&E and dark red on an MT stain. On the VVG stain, dermal elastic fibers were mildly increased in number multifocally, irregularly distributed throughout the dermis, and variably sized. The space between collagen fibers as well as the number and arrangement of dermal fibroblasts was similar between the affected and control dogs. The epidermis was of normal thickness in both the affected and control dog skin.

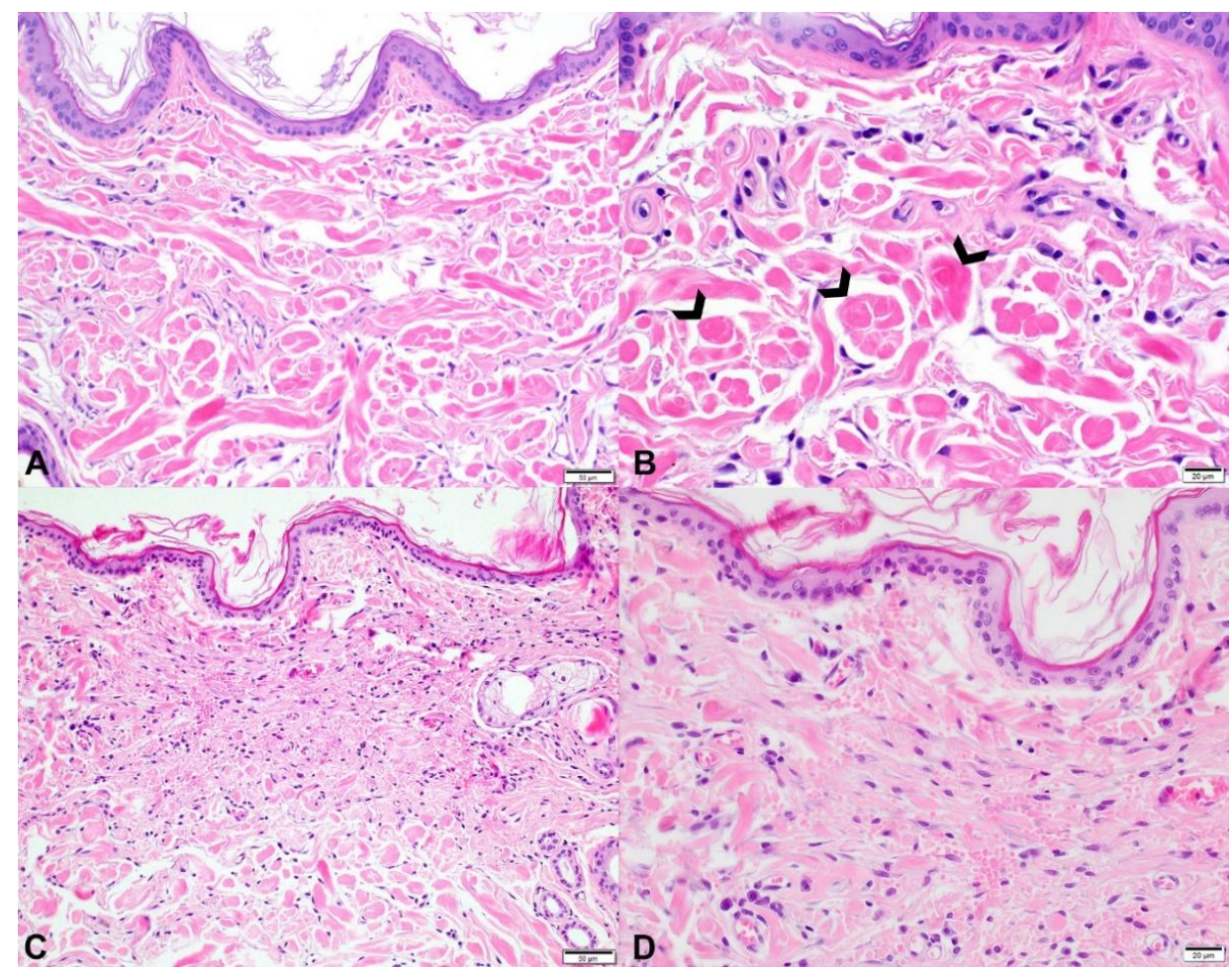

Figure 6. Comparison of histopathology findings in the skin of two dogs with Ehlers-Danlos syndromes (EDS)-like syndrome attributed to different mutations in the COL5A1 gene. (A,B): Photomicrographs of skin from the dorsum of the dog (case 1) with EDS-like syndrome attributed to a single nucleotide deletion and premature stop codon formation in the COL5A1 gene. Throughout the dermis, there is collagen fiber disorganization with great variation in fiber diameter, length, and staining intensity. $200 \times$ original magnification; bar $=50$ microns. (B): Collagen fibers frequently form variably sized, hypereosinophilic clumps (open arrowheads). 200× original magnification; bar $=50$ microns. (C,D): Photomicrographs of skin from one of the affected siblings with EDS-like syndrome attributed to a missense variant in the COL5A1 gene. (C): Underneath the unremarkable epidermis, abnormal collagen fibers are present in the superficial and mid dermis. Collagen fibers are very short, thin, wispy, and disorganized. Some broader, hypereosinophilic fibers are present. $200 \times$ original magnification; bar $=50$ microns. (D): In the upper dermis, horizontally aligned fibroblasts are present, indicating reparation of previous ruptures. In between the altered collagen fibers, there are numerous extravascular erythrocytes compatible with excessive hemorrhage. Compared to case 1 (panels A,B), large dermal collagen clumps are not present. 400× original magnification; bar $=20$ microns. 


\subsubsection{Cases 2 and 3}

Histological examination of the skin biopsies from these affected siblings revealed abnormal collagen fibers characterized by a severe variability in fiber diameter ranging from thin and wispy to normal thickness. In addition, there was a shortening of the fibers and fiber disarray. Abnormal fibers were outlined by increased numbers of fibroblasts. Space between fibers was wider than normal, and there was abundant hemorrhage, especially in the mid dermis. The dermis and the overlying epidermis were of normal thickness.

\subsection{Transmission Electron Microscopy}

The clinical and histological features discussed above suggested that all three cases might have a form of Ehlers-Danlos syndrome. In case 1, tissue was available for detailed ultrastructural analysis, and transmission electron microscopy of the dermis revealed abnormal fibril architecture that was also consistent with EDS. While in the control dermis, the collagen fibrils were highly organized and aligned with an approximately circular cross-sectional shape of uniform diameter (Figure 7A-D), the affected dog dermis had in addition to this normal fibril architecture a significant second heterogeneous population of disorganized collagen fibrils with large and/or irregular cross-sectional shapes (Figure 7E-H). This fibrillar disorganization has been widely reported in human EDS patient skin [20] and mouse models of EDS [19] and has also been reported in dogs [11]. Unfortunately, samples were not available from cases 2 and 3 for ultrastructural analysis of collagen fibrillar architecture.
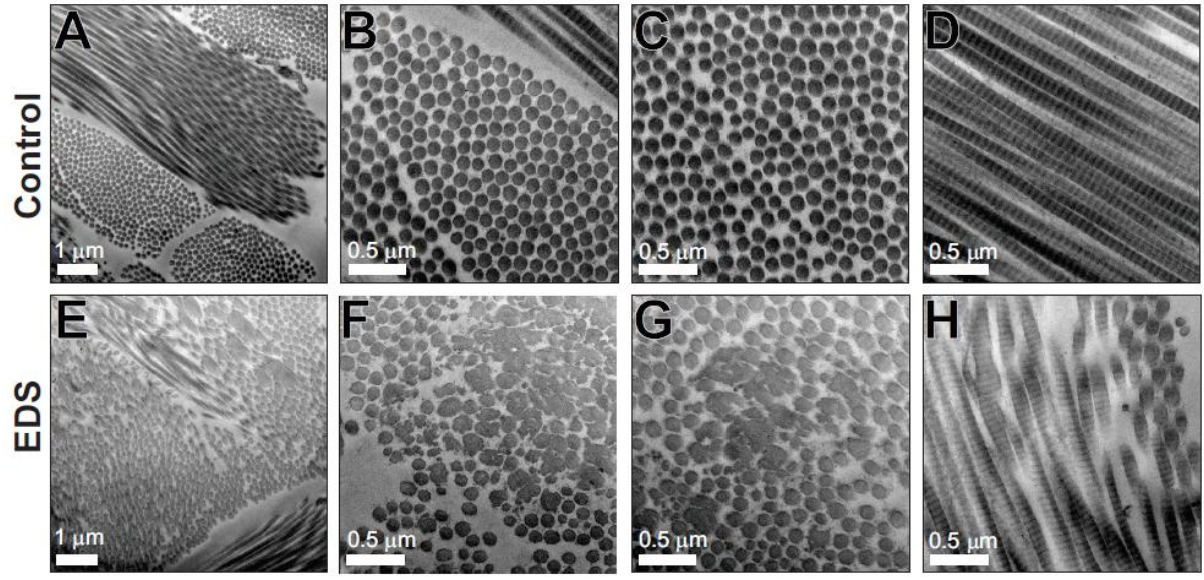

Figure 7. Structurally abnormal dermal collagen fibrils in Case 1. Transmission electron micrographs from the dermis of control (A-D) and Case 1 (EDS) (E-H). Control dermis contains well-organized collagen fibrils of uniform size and circular profile, while the EDS dermis contains two populations of fibrils. One fibril population is similar to the control dermis, whereas the other heterogeneous population contains large and structurally abnormal fibrils. Scale bars of $0.5 \mu \mathrm{m}$ or $1 \mu \mathrm{m}$ are shown.

\subsection{Biochemical Analysis of Dermis}

For case 1, dermal tissue was also available for biochemical analysis of the collagen composition. Extraction with isotonic neutral pH buffer (NSS), which removes the newly synthesized collagen species, accounted for only a small proportion of the total collagen extracted (Figure 8A, lanes 1-2) as did further extraction with $\mathrm{GuHCl}$ (lanes 3-4) or acetic acid (lanes 5-6), which extracts newly cross-linked collagen matrix. Note that lanes 1-6 were loaded with much higher proportions of the extracts to achieve Coomassie-detectable bands (Figure 8A, see legend for loading details). In all these extracts, collagen I and collagen III were the most abundant collagens with no apparent quantitative differences between EDS and control. The pepsin extracts of the dermis (Figure 8A, lanes 7,8 ) released the more cross-linked collagenous matrix as evidenced by the abundance of type I cross-linked dimers ( $\beta$-components) and trimers ( $\gamma$-components). There was no detectable qualitative 
difference in collagen I and III ratios or collagen I cross-linking in these samples. Collagen V was partially purified to provide additional qualitative data on the pepsin-extracted $\alpha 1(\mathrm{~V})$ and $\alpha 2(\mathrm{~V})$ chains (Figure $8 \mathrm{~B})$. Both $\alpha 1(\mathrm{~V})$ and $\alpha 2(\mathrm{~V})$ chains had a normal electrophoretic migration excluding major structural mutations affecting the $\alpha$-chain size, or increased post-translational modifications, which could indicate helix-disrupting mutations.

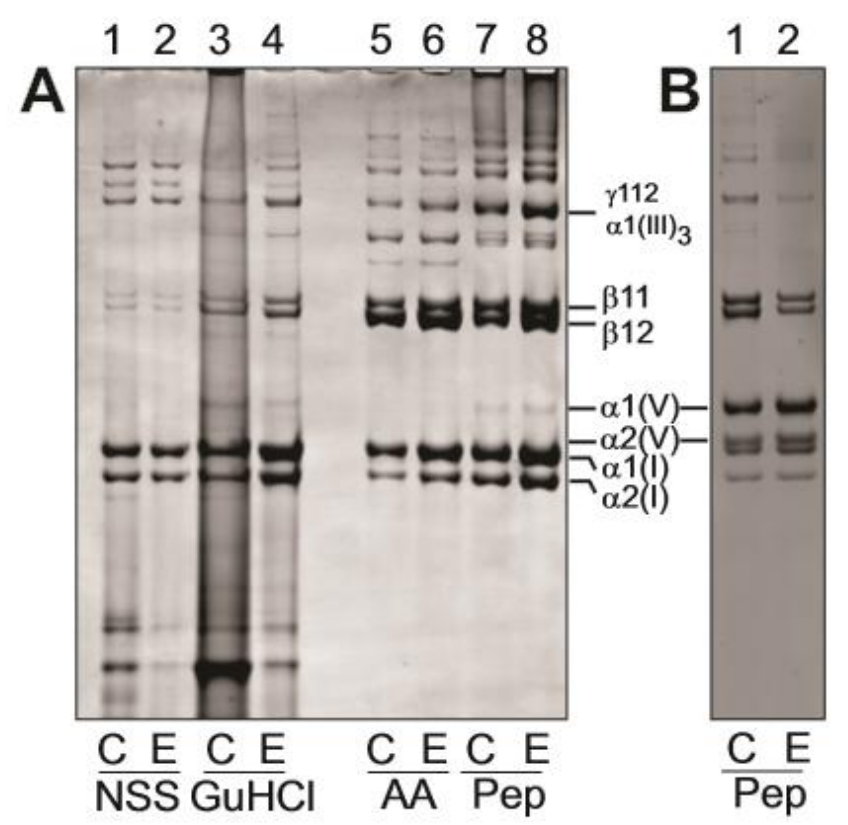

Figure 8. Dermal collagen extracellular matrix composition. Samples from control dermis (C) and EDS dermis (E) were extracted sequentially $0.15 \mathrm{M} \mathrm{NaCl}, 50 \mathrm{mM}$ Tris- $\mathrm{HCl}$ buffer, $\mathrm{pH} 7.4$ (neutral salt soluble, NSS), $4 \mathrm{M} \mathrm{GuHCl}, 0.5 \mathrm{M}$ acetic acid (AA) and digestion with pepsin (Pep) at $100 \mu \mathrm{g} / \mathrm{mL}$ to sequentially extract the successively more cross-linked and thus mature collagen matrix. (A) Extracts analyzed by SDS-polyacrylamide gel (3-8\%) electrophoresis; samples unreduced. (B) Pepsin extracts where collagen $\mathrm{V}$ chains were enriched by selective salt precipitation. Note that all samples were loaded to achieve a comparable staining intensity of control and EDS sample pair in each extract and do not reflect the relative amount of collagen between extracts. The vast majority of the collagen was found in the pepsin extract fractions ( $>95 \%$ ) of both the Control and EDS dermis. The migrations of collagen $\mathrm{V}$ $\alpha 1(\mathrm{~V})$ and $\alpha 2(\mathrm{~V})$ and the collagen I $\alpha 1(\mathrm{I})$ and $\alpha 2(\mathrm{I})$ chains and cross-linked $\alpha$-chain dimers ( $\beta 11$ and $\beta 12)$ and trimers $(\gamma 112)$ are shown. The disulfide-bonded collagen III trimer is denoted by $\alpha 1(\mathrm{III})_{3}$.

\subsection{Genetic Analyses}

\subsubsection{Case 1}

We sequenced the whole-genome of the affected Labrador Retriever at $\sim 18 \times$ coverage and called SNVs and short indels with respect to the canine reference genome (CanFam 3.1). The variants were compared to 356 control dogs from diverse breeds. We analyzed the data under two alternative models regarding the mode of inheritance: monogenic recessive or monogenic dominant. For the recessive model, we only considered variants at which the affected dog was homozygous for the non-reference allele, and none of the control dogs carried the non-reference allele. Hard-filtering resulted in four private protein-changing variants present in the affected dog (Table 1). None of these variants was located in a known candidate gene for Ehlers-Danlos syndrome. When filtering for heterozygous genotypes in the affected dog under the alternative dominant model, our automated pipeline detected 31 private protein-changing variants, among them a single nucleotide deletion in the COL5A1 gene. This variant, XM_022423936.1,c.3038delG, led to a shift in the reading frame and a premature stop codon p.(Gly1013ValfsTer260). 
Table 1. Homozygous and heterozygous private genetic variants in case 1 and case 2.

\begin{tabular}{ccc}
\hline Filtering Step & \multicolumn{2}{c}{ Number of Variants } \\
Case 1 & Case 2 \\
\hline $\begin{array}{c}\text { homozygous variants in the whole-genome } \\
\text { homozygous protein-changing variants in the } \\
\text { whole-genome }\end{array}$ & $2,791,220$ & $2,384,739$ \\
$\begin{array}{c}\text { homozygous private protein-changing variants } \\
\text { homozygous private protein-changing variants in } \\
\text { candidate genes }\end{array}$ & 12,121 & 11,065 \\
\hline $\begin{array}{c}\text { heterozygous variants in the whole-genome } \\
\text { heterozygous protein-changing variants in the } \\
\text { whole-genome }\end{array}$ & 0 & 9 \\
$\begin{array}{c}\text { heterozygous private protein-changing variants } \\
\text { heterozygous private protein-changing variants in } \\
\text { candidate genes }\end{array}$ & 10,310 & 0 \\
\hline
\end{tabular}

The heterozygous genotype at this variant in case 1 was confirmed by Sanger sequencing, and the genotypes of the parents were determined (Figure 9). In the affected dog, the ratio of wildtype:mutant allele was $\sim 1,1$. The sire of case 1 was homozygous wildtype. In contrast, the wildtype:mutant allele ratio was $\sim 4,1$ in DNA isolated from the blood of the affected dog's dam, indicating that the mutation event had occurred in the dam and that she was a gonosomal mosaic.

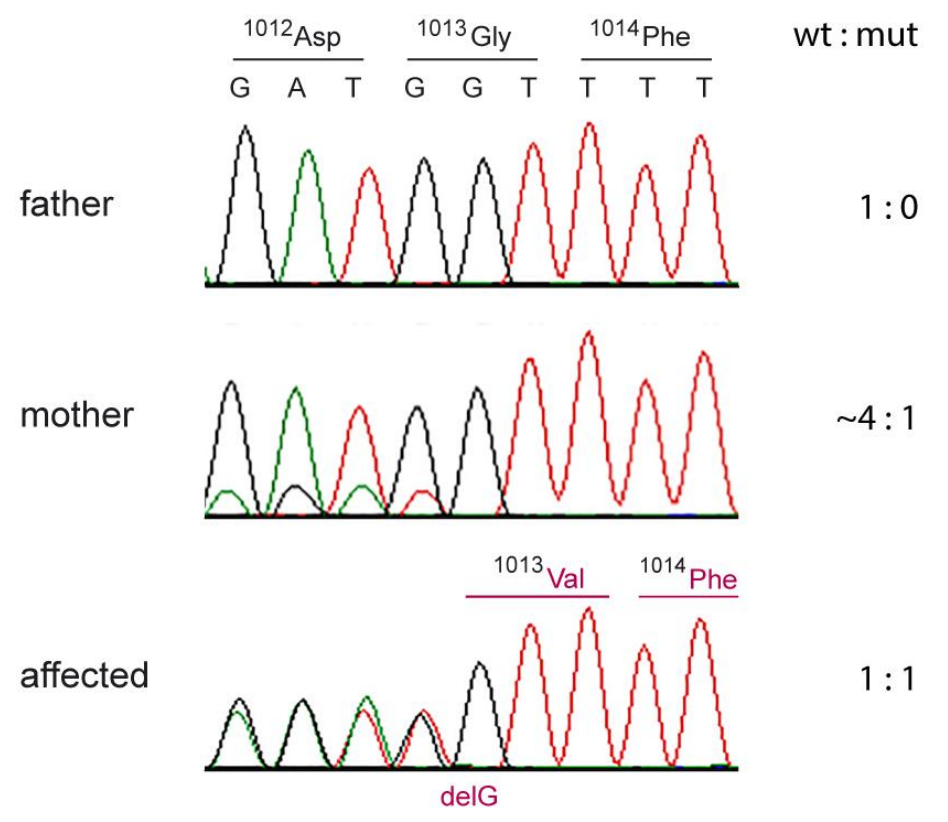

Figure 9. Sanger electropherograms illustrating the c.3038delG variant in case 1 and its parents. The sequence reads were obtained with a primer in reverse complementary orientation. While the affected dog shows a 1,1 ratio of the two alleles indicating a heterozygous genotype, the skewed allele ratio in the mother suggests somatic mosaicism. The mutant allele in the mother must have been present in white blood cells and her germline. This confirms that the c.3038delG variant arose by a de novo mutation event during the development of the mother.

\subsubsection{Cases 2 and 3}

We sequenced the whole-genome of case 2 at $~ 25 \times$ coverage. As in case $1, \mathrm{SNV}$ and short indel variants were called and compared to 356 control dogs, considering both a recessive and a dominant mode of inheritance. Our automated pipeline detected nine homozygous protein-changing variants in the affected dog, but none of them in a functional candidate gene (Table 1). Hard filtering for private 
protein-changing variants present in the heterozygous state in case 2 resulted in 276 variants, including a missense variant in the COL5A1 gene, XM_022423936.1,c.4711G>A; XP_022279644.1,p.(Gly1571Arg). Sanger sequencing confirmed a heterozygous genotype at the variant in case 2 and the available affected full-sibling (case 3), and the absence of the variant in 493 dogs from 66 different breeds (Table S1). DNA from the parents was not available.

\section{Discussion}

The clinical presentation in all three cases was consistent with a form of EDS. The most common form of EDS in humans, hypermobile EDS (hEDS), is an autosomal dominant condition, though the underlying genetic mutation has not been identified. Classical EDS (cEDS) is similarly an autosomal dominant condition, and mutations in collagen $\mathrm{V}$ genes (COL5A1 and COL5A2) have been reported [40]. In 2017, Malfait et al. proposed the International Classification for EDS [29]. With recent developments in next-generation sequencing and the discovery of a number of genetic defects in collagen biosynthesis and structure, human EDS has been reclassified into 13 subtypes based on major and minor criteria. Diagnosis of cEDS in humans relies on fulfilling minimal criteria (Table 2) as follows: Major criterion (1) skin hyperextensibility and atrophic scarring; plus either major criterion, (2) generalized joint hypermobility and/or at least three minor criteria. Confirmatory molecular testing is obligatory to reach a final diagnosis.

Table 2. Classical Ehlers-Danlos syndromes (EDS)-clinical criteria.

\begin{tabular}{|c|c|}
\hline Major criteria & $\begin{array}{l}\text { 1. Skin hyperextensibility and atrophic scarring } \\
\text { 2. Generalized joint hypermobility }\end{array}$ \\
\hline \multirow{10}{*}{ Minor criteria } & 1. Easy bruising \\
\hline & 2. Soft, doughty skin \\
\hline & 3. Skin fragility (or traumatic splitting) \\
\hline & 4. Molluscoid pseudotumors \\
\hline & 5. Subcutaneous spheroids \\
\hline & 6. Hernia (history thereof) \\
\hline & 7. Epicanthal folds \\
\hline & 8. Complications of joint hypermobility (e.g., sprains, \\
\hline & luxation/subluxation, pain, flexible flatfoot) \\
\hline & 9. Family history of a first-degree relative who meets clinical criteria \\
\hline
\end{tabular}

In case 1, the affected dog met both major criteria. One minor criterion, skin fragility, was also noted in the case history. This animal was 9 months of age at the time of euthanasia-it is possible that had the animal survived into adulthood more of the minor criteria would have been met. Regardless, the diagnosis of cEDS, in this case, was confirmed by the demonstration of the $1 \mathrm{bp}$ deletion mutation introducing a premature stop codon in the COL5A1 gene.

In cases 2 and 3, the major criteria of skin hyperextensibility (case 2 and 3) and atrophic scarring (case 2) were met; however, joint hypermobility was not evaluated. However, two minor criteria (easy bruising and traumatic splitting of the skin) were fulfilled. The diagnosis of cEDS in case 1 was confirmed by the demonstration of the missense mutation of the COL5A1 gene. While a missense mutation was demonstrated in cases 2 and 3, we could not strictly confirm the diagnosis of EDS without meeting the clinical criteria. It is worth noting that these criteria are a human EDS classification system, and too few cases have been reported in the literature to develop such a classification system for other species.

Some confusion has existed as to terminology as ED-like syndrome in dogs has been previously termed cutaneous asthenia. The term cutaneous asthenia in humans is reserved for cases where abnormalities in both collagen and elastin (cutis laxa) exist. The cutis laxa syndromes are no longer included within the EDS classification system and have not been diagnosed in animals [18]. 
Histopathology is typically pursued when heritable connective tissue disorders are suspected in animals. Morphologic changes in the skin may be absent or subtle on light microscopy, with reported changes typically including abnormal collagen fibers (collagen dysplasia) that are widely separated, finer and/or paler than normal, and haphazardly arranged. An increase in dermal elastic fibers has been described as a rare histopathologic feature of hereditary collagen dysplasia [41], but a distinction between the various forms of hereditary connective tissue disorders cannot be made based on a morphologic assessment of the tissue. In most cases, the ultrastructural examination is required to confirm the collagen abnormality detected by light microscopy [41].

Histopathology of skin from the three affected dogs revealed dermal collagen dysplasia, with collagen fibers that varied in diameter and length and were haphazardly arranged. Case 1 had numerous, large collagen clumps throughout the dermis (Figure 6B), while this was not a prominent feature in cases 2 and 3. Case 1 also had an increase in dermal elastic fibers, multifocally; a VVG stain was not performed in cases 2 and 3 . Cases 2 and 3 had more dermal fibroblasts and more space between collagen fibers, but this was not a feature of case 1 . The dermal thickness of case 1 could not be compared to the control since the sections came from areas of the body with innate differences in dermal thickness, i.e., the back and abdomen, respectively. Cases 2 and 3 had normal dermal thicknesses.

Collagen protein analysis of case 1 showed a normal collagen composition of the EDS skin compared to the control skin, with predominantly collagen I and smaller amounts of collagen III. Both EDS and control samples had, as expected, small amounts of collagen V, mostly in the pepsin-extracted cross-linked collagen fraction. There was no evidence of differential solubility of any of the collagen fractions, suggesting that the incorporation of all collagens into the dermal extracellular matrix was not impeded by the collagen $\mathrm{V}$ mutation. Genetic analysis of case 1 demonstrated a heterozygous COL5A1 p.(Gly1013ValfsTer260) mutation. Because of the introduction of a downstream premature termination codon in the mutant allele, this would be expected to result in $\alpha 1(\mathrm{~V})$ mRNA instability due to nonsense-mediated mRNA decay [42] resulting in $\alpha 1(\mathrm{~V})$ protein haploinsufficiency. Since $\alpha 1(\mathrm{~V})$ is an essential component of all collagen $\mathrm{V}$ helical trimers, an overall reduction in collagen $\mathrm{V}$ protein would result. A limitation of our studies is that we were unable to extract mRNA from the frozen tissue samples available, or obtain cell lines from case 1 to directly demonstrate the nonsense-mediated mRNA decay of the mutant COL5A1 allele. Haploinsufficiency of COL5A1 has been demonstrated in mice and humans and since the ratio of collagen V:collagen I is important for correct collagen I fibrillogenesis, reduced collagen $\mathrm{V}$ is associated with the EM findings of disorganized collagen fibrils and the clinical phenotype of cEDS [19,21,43].

Genetic analysis of cases 2 and 3 revealed a missense variant in the COL5A1 gene, p.(Gly1571Arg). The predicted amino acid exchange occurs in the protein helical domain, and by analogy with helix glycine substitution mutations, in collagen I in osteogenesis imperfecta, collagen II in chondrodysplasias, and collagen III and V in Ehlers Danlos syndrome, would be expected to disrupt collagen helix formation and/or stability [44]. In addition, intracellular retention of this structurally abnormal collagen could result in endoplasmic reticulum stress, which can impact cellular function. In addition, the intracellular degradation of the mutant collagen by the cellular quality control machinery may lead to reduced secretion of collagen $\mathrm{V}$ containing the mutant $\alpha 1(\mathrm{~V})$, also resulting in reduced extracellular collagen $\mathrm{V}$ available to regulate collagen I fibrillogenesis. Furthermore, any secretion of the mutant collagen $\mathrm{V}$ may interfere with collagen interactions in the extracellular matrix [44]. Unfortunately, neither dermal tissues nor cells were available from these dogs to directly explore these pathological mechanisms.

Interestingly, in case 1, the variant was passed on from a clinically non-affected mosaic parent to the offspring. It is, however, unknown whether the mother was clinically completely normal or showed subtle signs of Ehlers-Danlos syndrome. The genetic analysis of case 1 and its parents confirmed the suspected de novo mutation event. Given that only one protein-changing de novo mutation event per generation is expected on average, this further strongly supports the causality of the variant for the EDS phenotype. We speculated that a similar independent de novo mutation event had happened in 
one of the parents of cases 2 and 3. Unfortunately, no DNA samples from their parents were available, and we could not verify this assumption.

Our results were also consistent with recent findings in a cat that was also affected by Ehlers-Danlos syndrome due to a de novo mutation in the COL5A1 gene [45]. A recent paper demonstrated an 8-week-old Doberman Pinscher puppy with EDS to carry a rare, homozygous C-to-T transition at position 2408978 on chromosome 11. This transition is predicted to alter the ADAMTS2 transcript (ADAMTS2,c.769C $>$ T) and represents a nonsense variant (p.Arg257Ter) [46]. Whole-genome sequencing of another mixed-breed dog with EDS revealed two missense variants in the TNXB gene, encoding the extracellular matrix protein tenascin $\mathrm{XB}$ as potential candidate causative variants for EDS [47]. To the authors' knowledge, no previous studies have shown genetic variants in the COL5A1 gene in the dog.

These studies show that whole-genome sequencing as part of a "precision medicine" approach can be a valuable tool to obtain a precise definition of the molecular lesion in domestic animal patients suspected to suffer from inherited disease.

\section{Conclusions}

To the best of our knowledge, we provided the first report of genetic variants in the COL5A1 gene that are associated with the clinical presentation of EDS in dogs. Molecular screening using whole-genome or whole-exome sequencing or the targeted resequencing of a gene panel that includes the known EDS candidate genes from other species is a viable tool in the diagnosis of EDS in dogs. As in humans, any molecular techniques should always be combined with the established clinical criteria to confirm a diagnosis of EDS.

Supplementary Materials: The following are available online at http://www.mdpi.com/2073-4425/10/10/731/s1. Table S1. COL5A1,c.4711G > A genotypes of 493 dogs from 66 different dog breeds.

Author Contributions: All authors have read and approved the manuscript, A.B. performed the genetic analysis and contributed to writing, J.F.B. was involved in biochemical analysis of tissue samples, study design, and contributed to writing the manuscript, S.R.L. was involved in biochemical analysis of tissue samples and contributed to writing the manuscript, E.H. conducted the transmission electron microscopy, S.G.M.K. was involved in interpretation of histology for case 1 and contributed to writing, M.Y. was involved in case management, tissue collection, and clinical images, A.R. identified the cases from Morocco and was involved in clinical management and sample acquisition, V.J. performed the bioinformatics processing of the whole-genome sequence data and handled sequence data deposition to the ENA, M.W. performed the histopathological analyses on the cases from Morocco and contributed to writing the manuscript, T.L. supervised the genetic analysis and contributed to manuscript writing, F.L.B. was involved in clinical case management, sample collection, and was a major contributor in writing manuscript.

Funding: This study was funded in part by a grant of the Swiss National Science Foundation (CRSII3_160738) for genomic analysis and interpretation of data and by funding to the Murdoch Children's Research Institute from the Victorian Government's Operational Infrastructure Support Program for sample collection and biochemical analysis.

Acknowledgments: The Next-Generation Sequencing Platform of the University of Bern is acknowledged for performing the whole-genome re-sequencing experiment and the Interfaculty Bioinformatics Unit of the University of Bern for providing computational infrastructure. We acknowledge the Dog Biomedical Variant Database Consortium (Gus Aguirre, Catherine André, Danika Bannasch, Doreen Becker, Brian Davis, Cord Dröegemüller, Kari Ekenstedt, Kiterie Faller, Oliver Forman, Steve Friedenberg, Eva Furrow, Urs Giger, Christophe Hitte, Marjo Hytönen, Vidhya Jagannathan, Tosso Leeb, Hannes Lohi, Cathryn Mellersh, Jim Mickelson, Anita Oberbauer, Jeffrey Schoenebeck, Kim Summers, Frank van Steenbeck, Claire Wade) for sharing whole-genome sequencing data from control dogs and wolves. We thank all owners for donating samples and additional data of their animals.

Conflicts of Interest: The authors declare no conflict of interest.

\section{References}

1. Solomons, B. Equine cutis hyperelastica. Equine Vet. J. 1984, 16, 541-542. [CrossRef] [PubMed]

2. Hegreberg, G.A. Animal model: Ehlers-Danlos syndrome in dogs and mink, canine cutaneous asthenia. Am. J. Pathol. 1975, 79, 383-386. [PubMed] 
3. Harvey, R.G.; Brown, P.J.; Young, R.D.; Whitbread, T.J. A connective tissue defect in two rabbits similar to the Ehlers-Danlos syndrome. Vet. Rec. 1990, 126, 130-132. [PubMed]

4. Minor, R.R.; Lein, D.H.; Patterson, D.F.; Krook, L.; Porter, T.G.; Kane, A.C. Defects in collagen fibrillogenesis casuing hyperextensible, fragile skin in dogs. J. Am. Vet. Med. Assoc. 1983, 182, 142-148. [PubMed]

5. Persechino, A.G.; Roperto, F.; de Capraris, D.; Troncone, A. Rilievi clinici ed istopatologici in una collagenopatia del cane riportabile alla sindrome di Ehlers-Danlos dell'uomo [Collagenopathy in a dog resembling Ehlers-Danlos syndrome of man. Clinical and histopathological features]. Acta Med. Vet. 1983, 29, 67-79.

6. Papparella, S.R.; Troncone, A. Sindrome di Elhers-Dan-los nel cane. Rilievi ultrastrutturali della cute [Elhers-Danlos syndrome in a dog. Ultrastructural findings of the skin]. Acta Med. Vet. 1988, 34, 207-213.

7. Rodriguez, F.; Herráez, P.; Monteros, A.E.D.L.; Calabuig, P.; Rodríguez, J.L. Collagen dysplasia in a litter of Garafiano shepherd dogs. Zent. Fur Vet. Reihe A 1996, 43, 509-512.

8. Barnett, K.C.; Cottrell, B.D. Ehlers-Danlos syndrome in a dog: Ocular, cutaneous and articular abnormalities. J. Small Anim. Pract. 1987, 28, 941-946. [CrossRef]

9. Paciello, O.; Lamagna, F.; Lamagna, B.; Papparella, S. Ehlers-Danlos-like syndrome in 2 dogs: Clinical, histologic, and ultrastructural findings. Vet. Clin. Pathol. 2003, 32, 13-18. [CrossRef]

10. Barrera, R.; Mane, C.; Duran, E.; Vives, M.A.; Zaragoza, C. Ehlers-Danlos syndrome in a dog. Can. Vet. J. 2004, 45, 355-356.

11. Bellini, M.H.; Caldini, E.T.E.G.; Scapinelli, M.P.; Simões, M.J.; Machado, D.B.; Nürmberg, R.; Simões, M. Increased elastic microfibrils and thickening of fibroblastic nuclear lamina in canine cutaneous asthenia. Vet. Dermatol. 2009, 20, 139-143. [CrossRef]

12. Rasch, S.N. Surgical and medical treatment of ocular disease in a dog with Ehlers-Danlos syndrome. Clin. Case Rep. 2017, 5, 880-886. [CrossRef]

13. Uri, M.; Verin, R.; Ressel, L.; Buckley, L.; McEwan, N. Ehlers-Danlos Syndrome Associated with Fatal Spontaneous Vascular Rupture in a Dog. J. Comp. Pathol. 2015, 152, 211-216. [CrossRef]

14. Ueda, K.; Kawai, T.; Senoo, H.; Shimizu, A.; Ishiko, A.; Nagata, M. Histopathological and electron microscopic study in dogs with patellar luxation and skin hyperextensibility. J. Vet. Med. Sci. 2018, 80, 1309-1316. [CrossRef] [PubMed]

15. Counts, D.F.; Byers Holbrook, K.A.; Hegreberg, G.A. Dermatosparaxis in a Himalyan cat. J. Investig. Dermatol. 1980, 74, 96-99. [CrossRef]

16. Sequeira, J.L.; Bandarra, E.P.; Figueiredo, L.M.A.; Eugenio, F.R.; Rocha, N.S. Collagen Dysplasia (Cutaneous Asthenia) in a Cat. Vet. Pathol. 1999, 36, 603-606. [CrossRef] [PubMed]

17. Holbrook, K.A.; Byers, P.H.; Counts, D.F.; Hegreberg, G.A. Dermatosparaxis in a Himalayan cat. II. Ultrastructural studies of dermal collagen. J. Investig. Dermatol. 1980, 74, 100-104. [CrossRef] [PubMed]

18. Scott, D.W.; Miller, W.H.; Griffin, C.E. Congenital and Hereditary Diseases. In Muller and Kirk's Small Animal Dermatology, 6th ed.; Scott, D.W., Miller, W.H., Griffin, C.E., Eds.; WB Saunders: Philadelphia, PA, USA, 2001; pp. 913-1003.

19. Wenstrup, R.J.; Florer, J.B.; Davidson, J.M.; Phillips, C.L.; Pfeiffer, B.J.; Menezes, D.W.; Chervoneva, I.; Birk, D.E. Murine Model of the Ehlers-Danlos Syndrome $\mathrm{COL}_{5} \mathrm{~A}_{1}$ haploinsufficiency disrupts collagen fibril assembly at multiple stages. J. Biol. Chem. 2006, 281, 12888-12895. [CrossRef]

20. Bouma, P. COL $5 \mathrm{~A}_{1}$ Exon 14 Splice Acceptor Mutation Causes a Functional Null Allele, Haploinsufficiency of alpha $1(\mathrm{~V})$ and Abnormal Heterotypic Interstitial Fibrils in Ehlers-Danlos Syndrome II. J. Biol. Chem. 2001, 276, 13356-13364. [CrossRef]

21. Symoens, S.; Syx, D.; Malfait, F.; Callewaert, B.; De Backer, J.; Vanakker, O.; Coucke, P.; De Paepe, A. Comprehensive molecular analysis demonstrates type $\mathrm{V}$ collagen mutations in over $90 \%$ of patients with classic EDS and allows to refine diagnostic criteria. Hum. Mutat. 2012, 33, 1485-1493. [CrossRef]

22. Wenstrup, R.J.; Langland, G.T.; Willing, M.C.; D'Souza, V.N.; Cole, W.G. A splice-junction mutation in the region of COL5A1 that codes for the carboxyl propeptide of pro alpha $1(\mathrm{~V})$ chains results in the gravis form of the Ehlers-Danlos syndrome (type I). Hum. Mol. Genet. 1996, 5, 1733-1736. [CrossRef] [PubMed]

23. De Paepe, A.; Nuytinck, L.; Hausser, I.; Anton-Lamprecht, I.; Naeyaert, J.M. Mutations in the COL5A1 gene are causal in the Ehlers-Danlos syndromes I and II. Am. J. Hum. Genet. 1997, 60, 547-554. [PubMed] 
24. Nicholls, A.C.; E Oliver, J.; McCarron, S.; Harrison, J.B.; Greenspan, D.S.; Pope, F.M. An exon skipping mutation of a type $\mathrm{V}$ collagen gene $\left(\mathrm{COL}_{5} \mathrm{~A}_{1}\right)$ in Ehlers-Danlos syndrome. J. Med. Genet. 1996, 33, 940-946. [CrossRef] [PubMed]

25. Richards, A.J.; Martin, S.; Nicholls, A.C.; Harrison, J.B.; Pope, F.M.; Burrows, N.P. A single base mutation in $\mathrm{COL}_{5} \mathrm{~A}_{2}$ causes Ehlers-Danlos syndrome type II. J. Med. Genet. 1998, 35, 846-848. [CrossRef] [PubMed]

26. Toriello, H.V.; Glover, T.W.; Takahara, K.; Byers, P.H.; Miller, D.E.; Higgins, J.V.; Greenspan, D.S. A translocation interrupts the $\mathrm{COL}_{5} \mathrm{~A}_{1}$ gene in a patient with Ehlers-Danlos syndrome and hypomelanosis of Ito. Nat. Genet. 1996, 13, 361-365. [CrossRef]

27. Birk, D.E. Type V collagen: Heterotypic type I/V collagen interactions in the regulation of fibril assembly. Micron (Oxf. Engl. 1993) 2001, 32, 223-237. [CrossRef]

28. Hausser, I.; Anton-Lamprecht, I. Differential ultrastructural aberrations of collagen fibrils in Ehlers-Danlos syndrome types I-IV as a means of diagnostics and classification. Qual. Life Res. 1994, 93, 394-407. [CrossRef]

29. Malfait, F.; Francomano, C.; Byers, P.; Belmont, J.; Berglund, B.; Black, J.; Bloom, L.; Bowen, J.M.; Brady, A.F.; Burrows, N.P.; et al. The 2017 international classification of the Ehlers-Danlos syndromes. Am. J. Med. Genet. C Semin. Med. Genet. 2017, 175, 8-26. [CrossRef]

30. Bateman, J.F.; Golub, S.B. Deposition and selective degradation of structurally-abnormal type I collagen in a collagen matrix produced by osteogenesis imperfecta fibroblasts in vitro. Matrix Biol. 1994, 14, 251-262. [CrossRef]

31. Niyibizi, C.; Fietzek, P.P.; van der Rest, M. Human placenta type V collagens. Evidence for the existence of an alpha 1(V) alpha 2(V) alpha 3(V) collagen molecule. J. Biol. Chem. 1984, 259, 14170-14174.

32. Li, H.; Durbin, R. Fast and accurate short read alignment with Burrows-Wheeler transform. Bioinformatics 2009, 25, 1754-1760. [CrossRef] [PubMed]

33. Li, H. A statistical framework for SNP calling, mutation discovery, association mapping and population genetical parameter estimation from sequencing data. Bioinformatics 2011, 27, 2987-2993. [CrossRef] [PubMed]

34. McKenna, A.; Hanna, M.; Banks, E.; Sivachenko, A.; Cibulskis, K.; Kernytsky, A.; Garimella, K.; Altshuler, D.; Gabriel, S.; Daly, M.; et al. The Genome Analysis Toolkit: A MapReduce framework for analyzing next-generation DNA sequencing data. Genome Res. 2010, 20, 1297-1303. [CrossRef] [PubMed]

35. Bai, B.; Zhao, W.M.; Tang, B.X.; Wang, Y.Q.; Wang, L.; Zhang, Z.; Yang, H.C.; Liu, Y.H.; Zhu, J.W.; Irwin, D.M.; et al. DoGSD: The dog and wolf genome SNP database. Nucleic Acids Res. 2015, 43, D777-D783. [CrossRef] [PubMed]

36. Van der Auwera, G.A.; Carneiro, M.O.; Hartl, C.; Poplin, R.; Del Angel, G.; Levy-Moonshine, A.; Jordan, T.; Shakir, K.; Roazen, D.; Thibault, J.; et al. From FastQ data to high confidence variant calls: The Genome Analysis Toolkit best practices pipeline. Curr. Protoc. Bioinform. 2013, 43, 11.10.1-11.10.33.

37. Cingolani, P.; Platts, A.; Wang, L.L.; Coon, M.; Nguyen, T.; Wang, L.; Land, S.J.; Lu, X.; Ruden, D.M. A program for annotating and predicting the effects of single nucleotide polymorphisms, SnpEff: SNPs in the genome of Drosophila melanogaster strain $\mathrm{w}^{1118}$; iso-2; iso-3. Fly 2012, 6, 80-92. [CrossRef]

38. Patterson, D.F.; Minor, R.R. Hereditary fragility and hyper-extensibility of the skin of cats. Lab. Investig. 1977, 37, 170. [PubMed]

39. Freeman, L.J.; Hegreberg, G.A.; Robinette, J.D. Ehlers-Danlos syndrome in dogs and cats. Semin. Vet. Med. Surg. Small Anim. 1987, 2, 221-227. [PubMed]

40. Joseph, A.W.; Joseph, S.S.; Francomano, C.A.; Kontis, T.C. Characteristics, Diagnosis, and Management of Ehlers-Danlos Syndromes: A Review. JAMA Facial Plast. Surg. 2018, 20, 70-75. [CrossRef]

41. Mauldin, E.; Peters-Kennedy, J. Integumentary System. In Jubb, Kennedy, and Palmer's Pathology of Domestic Animals, 6th ed.; Grant Maxie, M., St Louis, M.O., Eds.; Elsevier Inc.: Amsterdam, The Netherlands, 2016; pp. 542-546.

42. Fang, Y.; Bateman, J.F.; Mercer, J.F.; Lamande, S.R. Nonsense-mediated mRNA decay of collagen-emerging complexity in RNA surveillance mechanisms. J. Cell Sci. 2013, 126, 2551-2560. [CrossRef]

43. DeNigris, J.; Yao, Q.; Birk, E.K.; Birk, D.E. Altered dermal fibroblast behavior in a collagen V haploinsufficient murine model of classic Ehlers-Danlos syndrome. Connect Tissue Res. 2016, 57, 1-9. [CrossRef] [PubMed]

44. Bateman, J.F.; Boot-Handford, R.P.; Lamandé, S.R. Genetic diseases of connective tissues: Cellular and extracellular effects of ECM mutations. Nat. Rev. Genet. 2009, 10, 173-183. [CrossRef] [PubMed] 
45. Spycher, M.; Bauer, A.; Jagannatha, V.; Frizzi, M.; De Lucia, M.; Leeb, T. A frameshift variant in the $\mathrm{COL}_{5} \mathrm{~A}_{1}$ gene in a cat with Ehlers-Danlos syndrome. Anim. Genet. 2018, 49, 641-644. [CrossRef] [PubMed]

46. Jaffey, J.A.; Bullock, G.; Teplin, E.; Guo, J.; Villani, N.A.; Mhlanga-Mutangadura, T.; Schnabel, R.D.; Cohn, L.A.; Johnson, G.S. A homozygous ADAMTS2 nonsense mutation in a Doberman Pinscher dog with Ehlers Danlos syndrome and extreme skin fragility. Anim. Genet. 2019, 50, 543-545. [CrossRef] [PubMed]

47. Bauer, A.; de Lucia, M.; Leuthard, F.; Jagannathan, V.; Leeb, T. Compound heterozygosity for TNXB genetic variants in a mixed-breed dog with Ehlers-Danlos syndrome. Anim. Genet. 2019, 50, 546-549. [CrossRef]

(C) 2019 by the authors. Licensee MDPI, Basel, Switzerland. This article is an open access article distributed under the terms and conditions of the Creative Commons Attribution (CC BY) license (http://creativecommons.org/licenses/by/4.0/). 